\title{
Biomedical Applications of Nanomaterials: Diagnosis and Therapy of Thrombotic Disorders
}

\author{
D. Dash \\ Department of Biochemistry, Institute of Medical Sciences \\ Banaras Hindu University, Varanasi.
}

\begin{abstract}
We have employed unique properties of carbon-based as well as metallic nanomaterials to develop diagnostic / therapeutic devices targeted against thrombotic disorders. We have designed a novel graphene-based biosensor that can detect individuals with high coronary risk. Further, we describe an innovative strategy to ablate pathological thrombus in situ employing near-infrared laser-irradiated gold nanorods (photothermal therapy).
\end{abstract}

Keywords: Thrombus, biosensor, photothermal therapy, platelet-derived microparticles.

\section{Introduction}

Global status report of World Health Organization has categorized cardiovascular diseases, comprising of coronary artery disease, stroke, atherosclerosis and heart failure, as leading causes of death resulting in millions of casualties worldwide, followed by deaths due to diabetes. According to the WHO projection, the spectrum of cardiovascular diseases will be the largest cause of disability and death in India by 2020, thus replacing infectious diseases from that position. Although cardiovascular diseases affect people with advanced age, risk factors may appear relatively early. One such risk factor is circulating Platelet-derived Micro Particles (PMPs) in blood of patients suffering from myocardial infarction or peripheral arterial diseases (1). Elevated plasma levels of PMPs have been associated with enhanced risk of coronary heart disease in healthy individuals (2).
Platelet activation is central to the pathogenesis of arterial thrombosis. PMPs are membrane vesicles of less than $1 \mu \mathrm{m}$ diameter released from stimulated platelets (3). They play significant role in haemostatic response and their presence in circulation represents serious procoagulantrisk. Detection of PMPs at early stage of disease would aid in diagnosis, prevention and management of the pathology. Flow cytometry is the available technique for PMP detection but is associated with major drawbacks that include underestimation of PMP count, lack of universal standardization, time consuming experiments, high cost of the equipment and need for skilled operator. In contrast electrochemical biosensing stands far superior chance as an efficient and affordable tool for PMP detection.

'Biosensing' is an emerging concept based on amperometry, potentiometry and impedance

Correspondence : Dr. D. Dash, Professor \& Head, Department of Biochemistry, Institute of Medical Sciences, Banaras Hindu University, Varanasi-221005.

DR.V.R. KHANOLKAR ORATION delivered during NAMSCON 2016 at the All India Institute of Medical Sciences, Raipur. 
analysis, which detects transduction of biological events to electrochemical signals with high sensitivity (4). Nanoscale particles of gold, iron and silicon, as well as graphene and carbon nanotubes have been employed as immobilization matrices during fabrication of electrodes. Material immobilized on exposed surface of electrodes determines its specificity, which include enzymes, antibodies, proteins, aptamers or nucleotides depending upon the nature of the target. Although there has been attempt to detect microparticles using electrochemistry, the method does not differentiate between their cells of origin and types (5) and thus has limited medical application. Here we describe a simple, quick, sensitive and cost-effective method to detect PMPs circulating in blood of individuals with potential for point-of-care diagnostic at peripheral health care system. The novelty of this product lies in its ease of fabrication and detection method, as no electrochemical sensor has yet been reported which could facilitate quick screening and diagnosis of individuals at 'high-risk' for developing cardiovascular diseases, eliminating requirement of high end lab facilities and experienced technicians as needed in current PMP detection procedures.

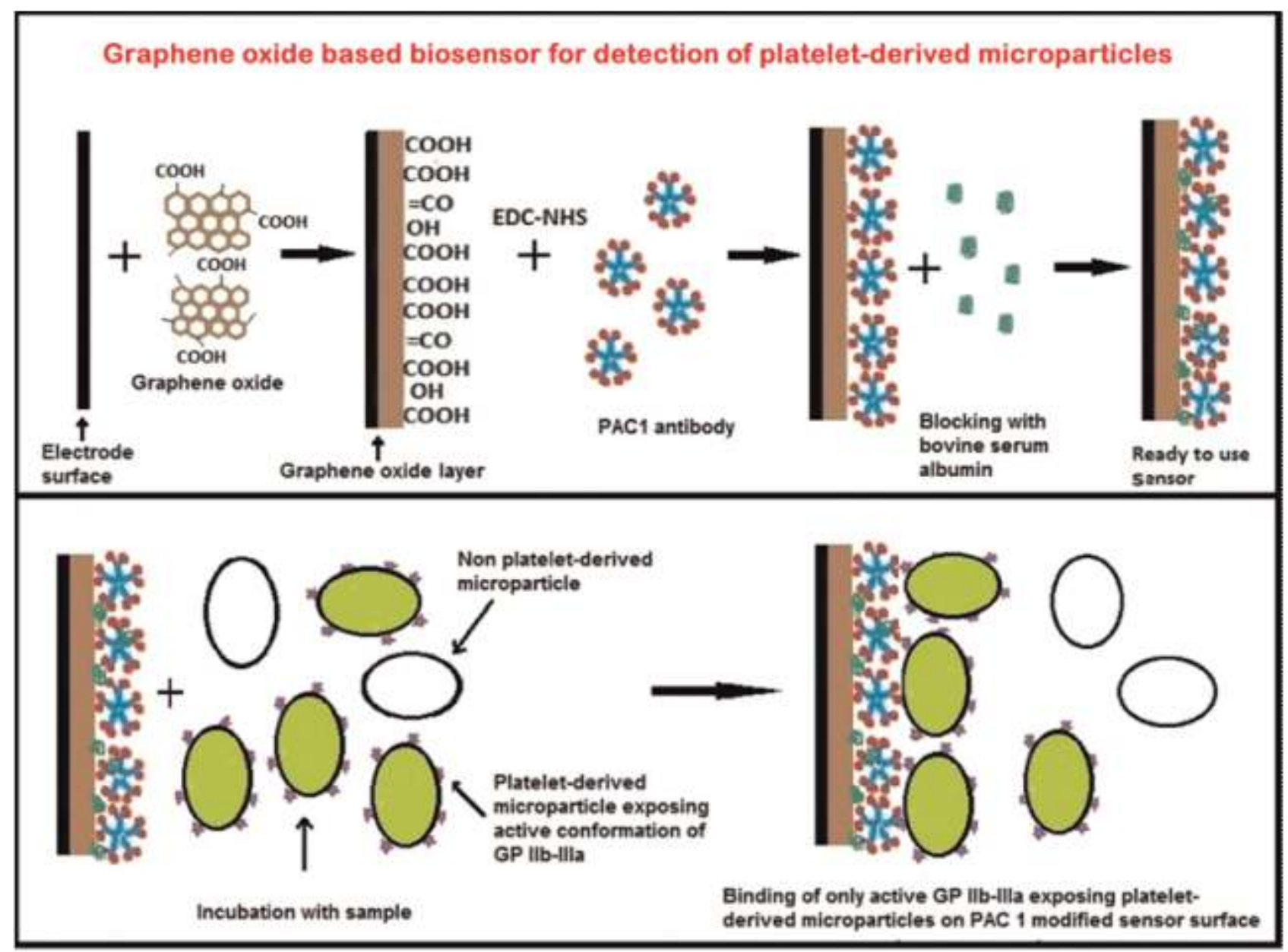

Fig. 1. Schematic design of nano-biosensor for detection of PMPs depicting stepwise immobilization of GO and PAC1 antibody on electrode surface. Subsequent incubation of coated electrode with sample resulted in binding of platelet-derived microparticles, bearing active conformation of integrins $\alpha_{\mathrm{II}} \beta_{3}$, to the sensor surface, which can be detected by impedance analysis. 
We have designed a graphene oxide-based electrochemical biosensor for detection of PMPs, a major risk factor for arterial prothrombotic pathologies like acute myocardial infarction and ischemic stroke. Electrodes were fabricated with immobilized layers of graphene oxide and a specific antibody targeted against active conformation of integrin $\alpha_{\mathrm{II}} \beta_{3}$ on PMP surface. Results showed progressive rise in impedance in Nyquist plots with increasing number of PMPs in analyte. The sensor was highly specific for PMPs and did not identify microparticles originating from other cells. Blood obtained from patients diagnosed with acute myocardial infarction exhibited significantly higher values of impedance, consistent with larger number of circulating PMPs in these patients, as compared to samples from healthy individuals, thus validating biosensor as a specific, sensitive, label-free and cost-effective tool for rapid point-of-care detection of PMPs at bedside. Our biosensor is most ideal for mass population screening programs at periphery-level healthcare units with limited resources. It is aimed at early detection of individuals having higher imminent cardiovascular risk, as well as for routine analysis, which in turn would contribute to better management and survival of screened 'high-risk' subjects $(6,7)$.

Despite major scientific advances, noncommunicable diseases like acute myocardial infarction, cerebral ischemic stroke, and deep vein thrombosis, underpinned with vascular blockage mediated by thrombus, remain major causes of death, and morbidity across the globe, incidence of which far exceeds that of cancer. Medication with fibrinolytic agents (tissue plasminogen activator, or tPA, streptokinase, and urokinase) are the widely used clinical practice that involves activation of plasminogen leading to proteolytic degradation of polymerized fibrin clot. However, fibrinolytic therapy is frequently associated with serious life-threatening complications like severe haemorrhage, embolism, haemorrhagic stroke, and reperfusion arrhythmias, and requires consistent physician supervision, and monitoring. Parenteral, or oral therapy would lead to generalized effect as it is not directed at localized clot, which, therefore, is another major limitation of this therapy. In this study, we have exploited biocompatibility, and photothermal attributes of NIR-active materials like gold nanorods (8) to dissolve fibrin clots at site of lesion, and restore lumen patency. The so-called photothermal therapy has been widely suggested as an anti-cancer measure to ablate solid tumours. This is the first report on application of photothermal therapy as an anti-thrombotic measure. The serine protease thrombin proteolytically degrades fibrinogen, the soluble blood protein component, to fibrin. Fibrin monomers are held together by non-covalent bonds constituting the nascent insoluble thrombus, which are further cross-linked by transglutaminase action of clotting factor XIII. Subjecting thrombus to photothermal heating would lyse non-covalent interactions, resulting in loosening, and downsizing of clot. Force of arterial, or venous fluid shear would disperse the remnants of loosened clot leading to drop in local concentration of polymerized fibrin, and relief from occlusion. Clot rarefication would also facilitate permeation of thrombolytics inside the thrombus mass.

Fibrinolytic therapy for arterial or venous thrombotic disorders warrants systemic administration of thrombolytics like streptokinase, which is associated with serious bleeding complications. In this study, we have provided proof-of-concept of photothermal ablation of thrombus Thrombi were generated in vitro either from purified fibrinogen or from plasma, or in vivo in murine blood vessels. Gold nanorods were added on fibrin-rich clots in vitro or targeted towards thrombi in situ in mice, followed by irradiation with a $808 \mathrm{~nm}$ nearinfrared laser source at power density of 1.05 $\mathrm{W} / \mathrm{cm}^{2}$. Local rise in temperature (up to $55-65^{\circ} \mathrm{C}$ ) was detected with an infrared thermal camera that leads to nearly $15 \%$ lysis of clot. This is the first report on application of photothermal therapy as an anti-thrombotic 


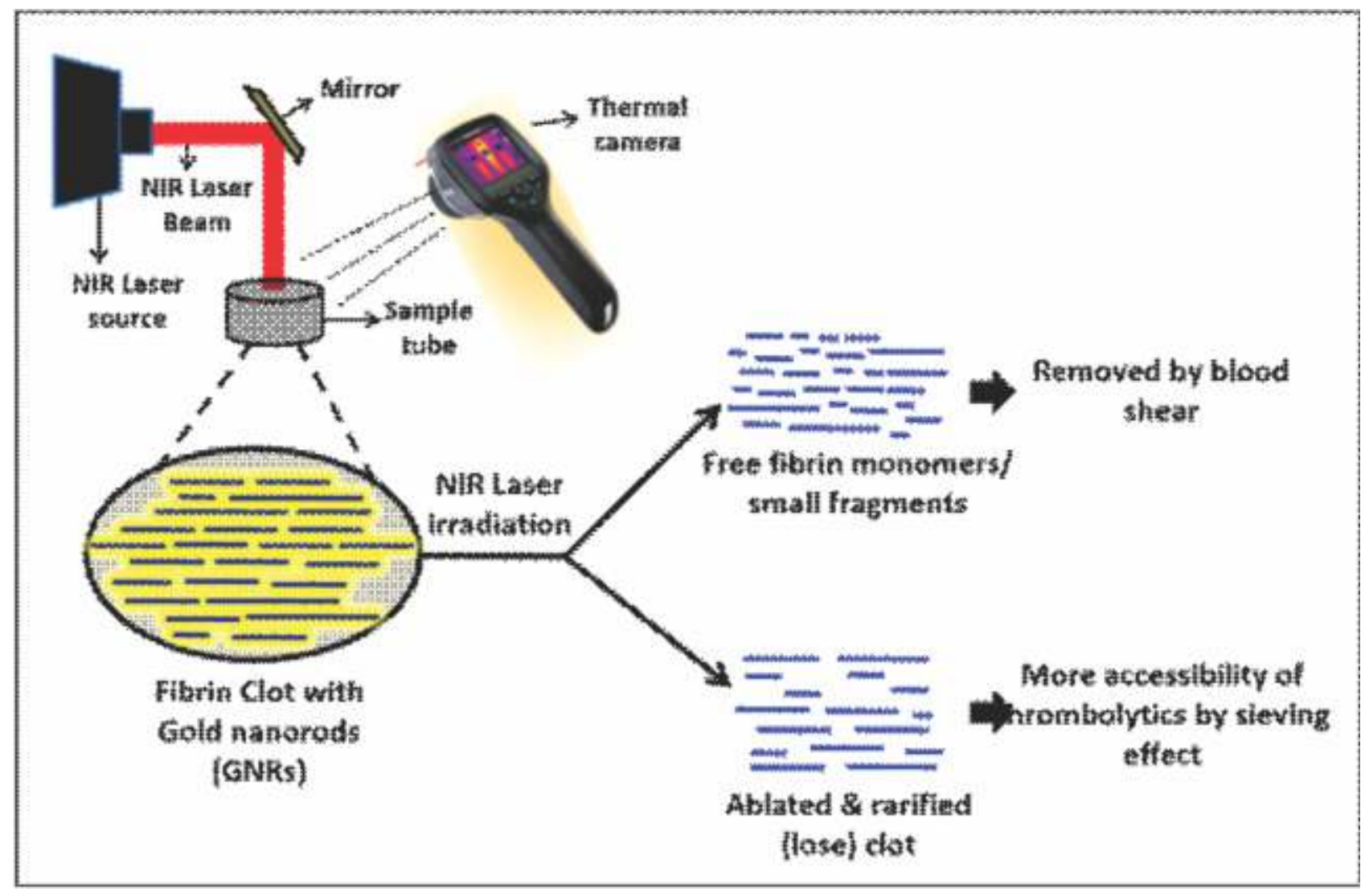

Fig. 2: Targeted photothermal therapy, when synergized with chemotherapy/thrombolytics at sub-therapeutic doses, exhibits great potential for the lysis of pathological clots with effective minimization of the life-threatening side effects and off-target adverse complications associated with existing therapy.

This introduces the possibility of smart and safe multi modal thrombolytic regimen in the future.

measure. Remarkably, addition of streptokinase has a multimodal additive effect in accelerating the photothermal lysis of thrombi (up to $40 \%$ ) even at a dose significantly lower (by 30 to 50 times) than therapeutic concentration, thus minimizing life-threatening side effects and adverse complications. This combinatorial approach has great potential in bringing about lysis of pathological clots that can effectively overcome the drawbacks of existing therapies $(9,10)$. Thus, the synergism between photothermal therapy and chemotherapy with reduced bleeding risk opens possibility of a smart and safe multimodal thrombolytic regimen for future.

\section{Acknowledgements}

This research was supported by grants received from the Department of Science and Technology (DST), Department of Biotechnology (DBT), Indian Council of Medical Research (ICMR), and the Council of Scientific and Industrial Research (CSIR), Government of India. Author thankfully acknowledges DST-FIST program and Tata Innovation Fellowship grant received from DBT. 


\section{References}

1. van der Zee PM, Biro E, Ko Y, et al (2006). P-selectin- and CD63-exposing platelet microparticles reflect platelet activation in peripheral arterial disease and myocardial infarction. Clin Chem 52: 657-664.

2. Ueba T, Nomura S, Inami N, et al (2010). Plasma level of platelet-derived microparticles is associated with coronary heart disease risk score in healthy men. $J$ Atheroscler Thromb 17: 342-349.

3. Siljander PR (2011). Platelet-derived microparticles - an updated perspective. Thromb Res 127 (Suppl 2):S30-S33.

4. Higgins IJ, Lowe CR (1987). Introduction to the principles and applications of biosensors. Philos Trans $R$ Soc Lond B Biol Sci 316: 3-11.

5. Lvovich V, Srikanthan S, Silverstein RL (2010). A novel broadband impedance method for detection of cell-derived microparticles. Biosens Bioelectron 26: 444-451.
6. Kailashiya J, Singh N, Singh SK, Agrawal V, Dash D (2015). Graphene oxide-based biosensor for detection of platelet-derived microparticles: a potential tool for thrombus risk identification. Biosens Bioelectron 65: 274-280.

7. Patent No. 1959/DEL/2013, dated 02.07.2013.

8. Tsai MF, Chang SH Gilbert, Cheng FY, et al (2013). Au nanorod design as lightabsorber in the first and second biological near-infrared windows for in vivo photothermal therapy. ACS Nano 7: 5330-5342.

9. Singh N, Varma A, Verma A, Maurya BN, Dash D (2016). Relief from vascular occlusion using photothermal ablation of thrombus with a multimodal perspective. Nano Res 9: 2327-2337.

10. Patent No. 3168/DEL/2014, dated 03.11.2014. 